\title{
Perspective: Differential Susceptibility to the Environment and Borderline Personality Disorder
}

\author{
Charlie Rioux, BSc, ${ }^{1}$ Jean R. Séguin, PhD, ${ }^{2}$ Joel Paris, MD $^{3}$ \\ ${ }^{1}$ Department of Psychology, Université de Montréal; CHU Ste-Justine Research Centre \\ ${ }^{2}$ Department of Psychiatry, Université de Montréal; CHU Ste-Justine Research Centre \\ ${ }^{3}$ Department of Psychiatry, McGill University; Department of Psychiatry, Jewish General \\ Hospital
}

Correspondence: Charlie Rioux, CHU Ste-Justine Research Centre, Université de Montréal, 3175 Chemin de la Côte Sainte-Catherine, Montréal (Québec), Canada, H3T 1C5. Tel: 514-3454931 ext. 7472; Fax: 514-345-2176; Email: charlie.rioux@umontreal.ca.

Conflicts of Interest and Source of Funding: All authors report no conflict of interest. This article was supported by the Fonds de la Recherche du Québec - Santé through a scholarship to $\mathrm{CR}$. The funding source had no role in writing the manuscript, or the decision to submit the paper for publication.

Accepted manuscript published in Harvard Review of Psychiatry - Full reference:

Rioux C., Séguin, J. R. \& Paris, J. (2018). Differential susceptibility to the environment and borderline personality disorder. Harvard Review of Psychiatry. 26(6), 374-383. doi: 10.1097/HRP.0000000000000182. 


\title{
Perspective: Differential Susceptibility to the Environment and Borderline Personality Disorder
}

\begin{abstract}
Evolutionary models of psychopathology can shed light on gene-environment interactions. Differential susceptibility to the environment means that heritable traits can have positive or negative effects depending on environmental context. Thus, traits that increase risk for mental disorders when the environment is negative can be adaptive when the environment is positive. This model can be applied to borderline personality disorder (BPD), viewing predictors such as emotional dysregulation and impulsivity, as temperamental variations leading to negative effects in an unfavorable environment, but to positive effects in a favorable environment. This model may also be useful in conceptualizing the mechanisms of effective therapy for BPD.
\end{abstract}

Keywords: Borderline Personality Disorder, Human Development, Environment, GeneEnvironment Interaction, Family 


\section{Perspective: Differential Susceptibility to the Environment and Borderline Personality Disorder}

\section{Basic Principles of Evolutionary Psychiatry}

Since natural selection shapes mind and brain, evolutionary theory can make a major contribution to the understanding of the causes of psychopathology. ${ }^{1}$ The heritability of mental disorders, and of personality traits, has been strongly supported by the findings of behavior genetic research..$^{2,3}$ Twin studies show that virtually all traits that affect behavior, emotion, and cognition have a genetic component that accounts for about half the variance in the outcome, while an environmental component accounts for the other half of the variance..$^{2-4}$

Traits that underlie variations in susceptibility to medical and psychiatric illnesses are also subject to natural selection. ${ }^{5}$ But selection does not necessarily remove these variations from the gene pool. Moreover, not all heritable traits produce optimal outcomes, and some that are preserved in the population seem at first sight to be maladaptive. One explanation could be that these characteristics become problematic under one set of circumstances, but advantageous under another set of circumstances. ${ }^{6}$ In this model, traits associated with illness are not just markers for vulnerability: what is inherited is not necessarily susceptibility to disorder, but tendencies to respond to the environment in specific ways. Thus, depending on whether the rearing environment is stressful or supportive, personality trait variations can lead to positive or negative outcomes. $^{7}$

One example concerns one of the most common of all mental illnesses, clinical depression. While severe depression is maladaptive, milder forms may not be, but can reflect an adaptive response to defeat that is associated with greater resilience. ${ }^{8}$ There may also be a link 
between depression and other adaptive traits; for example, several studies suggest that mood disorders can be associated with higher levels of creativity. ${ }^{9-12}$

Personality disorders provide an even better example. These conditions can be understood as pathological amplifications of normal variations in personality. ${ }^{13}$ For example, compulsive traits can be associated with persistence and reliability, and narcissistic traits can be associated with ambition and optimism. ${ }^{14}$ Thus, these traits can be adaptive under some circumstances, while a personality disorder can emerge from interactions with an adverse environment. $^{15}$

\section{Differential Susceptibility to the Environment}

The social environment can have a significant influence on development. On the one hand, adverse environments, which are characterized by stressors such as child maltreatment, insensitive parenting or negative life events, are associated with a higher occurrence of mental health symptoms. On the other hand, positive environments, characterized by supportive and enriching experiences as well as by the absence of adversity, can be beneficial and are associated with a lower occurrence of mental health symptoms.

According to Life history theory, biological traits such as growth rate, age, body size at sexual maturation, number of offspring, and length of lifespan are modeled by environmental conditions. At a psychosocial level, children raised in positive environments would see the world as safe, and thus pursue slower life history strategies, which include maturing and reproducing later and forming stable long-term relationships. Life history theory also suggests that a less optimal development resulting from adverse environments could also be adaptive from an evolutionary standpoint. Accordingly, children raised in adverse environments would see the 
world as unpredictable, and thus pursue faster life history strategies, which include earlier maturation, sexual activity and reproduction. ${ }^{16-18}$ But faster life histories could also be maladaptive and associated with psychopathology (e.g., emotional dysregulation, impulsivity, lack of trustworthiness). ${ }^{19,20}$ In a similar manner, it has been suggested that the brain may be conditioned by an adverse environment early in life in such a way as to allow the individual to prepare and adapt to life-long stress or deprivation. ${ }^{21,22}$ However, the view that the environment alone can be considered as a single influence on the development of psychiatric disorders has shown its limits, and one must consider its interaction with individual factors. Indeed, individuals may vary in the degree to which they are influenced by the environment, and thus a personenvironment interactive framework may help to fully understand and integrate the associations between the environment, life history strategies, brain development and psychiatric disorders. Differential susceptibility to the environment ${ }^{23,24}$ is a construct that illuminates some of the mechanisms behind person-environment interactions in psychopathology. The theory has been proposed as an alternative to the well-known diathesis-stress framework. ${ }^{25}$ Indeed, the diathesis-stress model tends to be used in both clinical and research settings to explain interactions between environmental and individual characteristics and suggests that more "vulnerable" individuals will develop mental disorders when exposed to adverse environments, while more "resilient" individuals will remain healthy regardless of their environment (see Figure 1). ${ }^{25}$ In contrast, the differential susceptibility theory, based on evolutionary biology, proposes that "susceptible" individuals will do worse than average when exposed to an adverse environment, but better than average in a positive environment (see Figure 1). ${ }^{23}$ Thus, these individuals can be considered more malleable when exposed to both positive and adverse environmental influences, rather than only vulnerable to adverse environmental influences as the 
diathesis-stress model suggests. Because the same genetic potential can lead to a positive or negative outcome, this theory helps explain why alleles associated with both high and low susceptibility to pathology are retained in the gene pool. Indeed, having children with varying levels of susceptibility to rearing influences within the same family would be expected to maximize reproductive fitness. On the one hand, in an adverse environment, resilient children will not reap the costs of the negative environment and thus maximize reproductive fitness within that environment. On the other hand, in a positive environment, more susceptible children will reap the most benefits from the environment, which will maximize reproductive fitness. ${ }^{26}$ As Simpson and Belsky ${ }^{27}$ (p. 109) point out: “...differential susceptibility could be adaptive... if a parent's attempt to "prepare" his or her children for the future environment is mistaken due to inherent unpredictability of future conditions". Using a similar theoretical model, Boyce and Ellis ${ }^{28}$ suggest that "biological sensitivity to context" is a variable trait that regulates stress reactivity.

Empirical support for one or the other theoretical model can be obtained by careful examination and statistical testing of interaction patterns $\mathrm{s}^{29-32}$ - which was not done before the differential susceptibility model was highlighted as an alternative to the diathesis-stress model. Thus, older research examining person-environment interactions operated on the assumption that statistical interactions supported a diathesis-stress model and were consequently not designed to rule out the differential susceptibility hypothesis. Indeed, a growing number of re-analyzes of previous studies on person-environment interactions initially conducted within a diathesis-stress framework now reveal the conditions under which there is support for the differential susceptibility models. ${ }^{23,33}$ 
The concept of differential susceptibility has gained momentum in recent years and has stimulated a body of research with much evidence that now supports its complementarity to the diathesis-stress model in understanding developmental psychopathology. Dozens of studies providing support for the model have been reviewed elsewhere. ${ }^{23,33,34}$ Examples are given here to provide an idea of the wide range of the evidence supporting the model. Some studies have focused on observable characteristics as susceptibility factors, the most studied being temperament, which represents individual differences in behavior-influencing traits that appear early and are relatively stable across situations and time. ${ }^{35-37}$ Specific temperament dimensions are studied (e.g., impulsivity, inhibitory control, activity level, negative emotionality, harm avoidance), as well as overarching temperament profile, which are a cluster of temperamental dimensions, the most commonly used being the easy and difficult temperament profiles; ${ }^{33}$ a child with a difficult temperament usually has irregular eating and sleeping daily routines, withdraws from people and novel stimuli, is inflexible to changes in the environment, has high intensity responses and an irritable mood. ${ }^{38}$

Studies on interactions between temperament (both specific dimensions and overarching profiles) and the familial environment have showed support for differential susceptibility in the prediction of several developmental outcomes. For example, a longitudinal study examined interactions between impulsivity and coercive parenting (i.e., use of harsh physical and/or verbal discipline) and found that highly impulsive 6-year-old children were more vulnerable to high levels of coercive parenting than less impulsive children, but also benefited more from low levels of coercive parenting when predicting alcohol use at 15 years. ${ }^{39}$ Regarding overarching temperament profiles, a recent meta-analysis of research on the interaction between temperament and parenting practices within a differential susceptibility framework concluded that "children 
with a more difficult temperament (compared with those with a more easy temperament) were more vulnerable to negative parenting, but also profited more from positive parenting, supporting the differential susceptibility model. Differences in susceptibility were expressed in externalizing and internalizing problems and in social and cognitive competence" (p. 216). ${ }^{40}$ Of note, and possibly relevant to prevention and intervention, environmental influences outside of the family were also found to interact with temperament, supporting differential susceptibility. For example, highly disinhibited 7-year-old children had high levels of mental health symptoms at 13 years when teacher-child closeness was lower, but also lower mental health symptoms than more inhibited children when teacher-child closeness was higher. ${ }^{41}$

The same principle has been applied to other observable characteristics than temperament, notably perceptual sensitivity. Aron and Aron $^{42}$ found that some individuals are unusually sensitive, not only to life experiences, but also to sensory input, which they refer to as sensory-processing sensitivity or more generally as high sensitive personality. Studies have found that this trait that can be both positive or negative depending on environmental factors, ${ }^{23,43}$ suggesting that it could be a good indicator of susceptibility.

Gene-environment interactions have also been an important focus in differential susceptibility research, with most research focusing on the serotonin transporter linked polymorphic region (5-HTTLPR) and the dopamine receptor D4 gene (DRD4). Once again, research has found interactions with the familial environment. For example, interactions supporting the differential susceptibility model were found between 5-HTTLPR and child maltreatment in the prediction of antisocial behaviors ${ }^{44}$ and between DRD4 and maternal positivity in the prediction of prosocial behavior. ${ }^{45}$ Genes can also be indicators of sensitivity to environmental influences outside of the family. For example, one study found an interaction 
between peer behaviors and a genetic variation affecting the 5HTTLPR allele that supported the differential susceptibility model in the prediction of substance use. ${ }^{46}$ Another study found that an interaction between 5HTTLPR and racial discrimination predicted conduct problems, supporting differential susceptibility. ${ }^{47}$ Finally, a meta-analysis also found that carriers of risk genotypes benefit more from interventions changing the environment for the better than other individuals, providing experimental support for differential susceptibility. ${ }^{48}$

A last type of susceptibility factor examined in differential susceptibility research is physiological reactivity, including cardiovascular reactivity, sympathetic nervous system reactivity and parasympathetic nervous system reactivity. With the familial environment, interactions supporting the differential susceptibility model have been found, for example, when looking at cortisol reactivity and stressful family life events in the prediction of externalizing problems ${ }^{49}$ and when looking at respiratory sinus arrhythmia reactivity and marital conflict in the prediction of externalizing problems. ${ }^{50} \mathrm{With}$ other environmental factors, a study supported differential susceptibility with an interaction between mean arterial pressure and the teacherchild relationship in the prediction of mental health symptoms. ${ }^{41}$

\section{Relationship to Etiological Theories of BPD}

The etiology of borderline personality disorder (BPD) has often been viewed as shaped by an adverse childhood environment. However, behavior genetic studies ${ }^{51,52}$ have shown that only half of the variance affecting an outcome of BPD is accounted for by environmental factors, and the other half of the variance affecting this disorder is heritable. This suggests that interactions between genes and the environment may account best for the pathways to BPD. ${ }^{53}$ This model would parallel the model developed by Caspi and Moffit ${ }^{54}$ for antisocial behavior, 
i.e., that neither genetic vulnerability nor environmental adversity alone are sufficient to produce pathology, but that a combination of both factors carries a significant risk.

The core trait underlying BPD is emotion dysregulation. ${ }^{55}$ In BPD, emotions are highly susceptible to the social environment, leading to sharp peaks of reactivity to adverse events, with a longer time needed to return to normal levels. ${ }^{55}$ This key feature of BPD has been shown to be heritable. ${ }^{2}$ Emotional dysregulation reflects unusually high levels of trait neuroticism, i.e., elevated reactivity to life events. ${ }^{13}$ But BPD patients do not necessarily react negatively to all events, and are most likely to do so when faced with trigger stimuli such as interpersonal rejection ${ }^{56,57}$ or shame. ${ }^{58}$ Since their lives are often marked by the choice of unstable attachment figures, this helps explain why BPD patients often describe their emotional life as a "roller coaster".

The precise biological mechanisms behind the heritable vulnerability to BPD are unknown. Some of the possibilities under investigation include: variations in the activity of oxytocin levels that modulate the attachment system; ${ }^{59,60}$ dopaminergic regulation of reward systems related to positive emotionality, as well as serotonergic regulation of mood and impulsivity related to emotional dysregulation. ${ }^{61}$ Variations in the activity of all these neurotransmitters seem to be associated with differential susceptibility to the environment. ${ }^{7}$

These theories, based mainly on neurotransmitter systems, may be too simple. Complex traits are not associated with single brain systems or single variations in neurochemistry, but with interactions between multiple systems. ${ }^{62}$ This is consistent with the finding that genome-wide association studies have generally reported variations of a very large number of alleles that are associated with most mental disorders. ${ }^{63}$ In BPD, while there have been many theories about its neurobiology, no biomarkers have been shown to have a consistent or specific relationship to the 
disorder. ${ }^{64}$ In any case, biological mechanisms for vulnerability can only be understood in the context of gene-environment interactions..$^{53}$

The literature on childhood adversity as a risk factor for BPD can also be understood as consistent with gene-environment interactions. The most common childhood adversities reported by BPD patients include dysfunctional families, sexual and/or physical abuse, and emotional neglect. ${ }^{65}$ However, none of these experiences, by themselves, predict the development of a mental disorder or a personality disorder. Even in the face of serious adversity, most children are resilient to adverse experiences. For example, it is rare for sibling pairs in which one has BPD to develop the same disorder ( $5 \%$ of cases), even when they experience the same problematic family environment. ${ }^{66,67}$ Instead, non-BPD siblings have a very different temperament, as measured by personality trait profiles. Once again, the explanation lies in gene-environment interactions. A review of studies that have specifically examined gene-environment interactions in $\mathrm{BPD}^{53}$ concluded that some evidence points to significant interactions between genes and environmental influences such as divorce/break-up, violent assault, sexual assault, and job loss. They also concluded that genes influencing borderline features increase the likelihood of being exposed to these adverse life events.

\section{Differential Susceptibility and the Traits Underlying BPD}

As previously described, the model of differential susceptibility to the environment differs from classical models of diathesis-stress. ${ }^{23,25}$ It hypothesizes a relationship between genes and environment that is not simply additive or subtractive, but one that determines how environmental factors shape pathological outcomes depending on an individual's plasticity to the environment. 
Many clinical features of BPD, such as severe mood instability and self-harm, are maladaptive. Moreover, BPD reduces fertility and seriously shortens the life span. ${ }^{68}$ One might therefore ask why this disorder has not been selected out of the population. But if the traits that put individuals at risk of developing BPD actually reflect differential susceptibility to the environment, they could lead to BPD in adverse environments, but also be adaptive in a positive environment. This differential susceptibility to environmental influences is highlighted by a metaphor differentiating between "orchid" and "dandelion" children. ${ }^{28,69}$ According to this metaphor, most children can, like dandelions, flourish in a wide range of environments. However, children with a trait that put them at risk for BPD are, like orchids, more dependent on their environment - they will not adapt when neglected, but they can flourish and surpass their peers when they have access to sufficient levels of psychological "nutrients". This hypothesis has not yet been directly tested with BPD as an outcome, but it could be.

Some traits associated with a higher risk of developing BPD or higher levels of BPD symptoms could lead to either positive or negative outcomes. A good example concerns emotional dysregulation. This core trait has been widely researched in BPD, using self-report scales $^{70,71}$ and event-contingent recording methods providing a more immediate self-assessment of emotional response. ${ }^{72,73}$ But less attention has been paid to the possibility that higher levels of emotional reactivity, which is likely at the root of emotional dysregulation, can sometimes be helpful and adaptive. Thus, a highly reactive person could experience not only intense negative feelings, but also more positive emotions. For example, while emotional reactivity is associated with externalizing disorders, longitudinal studies show that it does not necessarily produce psychopathology ${ }^{74}$ Moreover, people who are emotional and extraverted tend to be attractive to others. ${ }^{75}$ Interestingly, emotional reactivity is already seen early in development through 
children's temperament, with the temperament trait of negative emotionality representing a proneness to negative emotional experiences such as frustration, fear and shyness. ${ }^{76}$

Developmental studies have shown that this temperament trait interacts with the environment, supporting differential susceptibility in the prediction of externalizing problems ${ }^{77}$ and executive function, ${ }^{78}$ which could be explained by an increase in the role of sensitive parenting and parentchild mutuality for children high on negative emotionality. Indeed, it has been suggested that more skillful mothers may be more responsive to their child's emotional cues and thus provide adequate guidance to support the development of their child's regulatory skills. ${ }^{79}$ A parent-child relationship promoting self-regulatory capacity may be particularly consequential for children high on negative emotionality, with a particularly sensitive and responsive parenting style leading to superior developmental outcomes. ${ }^{77}$ As temperamental negative emotionality has already been shown to be associated with BPD,${ }^{80}$ it could be tested as an early susceptibility factor for later BPD symptoms.

Impulsivity, the second core trait behind BPD, ${ }^{81}$ manifested by self-harm, parasuicidal actions, and substance use, is the feature that brings most patients to clinical attention. It has also been shown that the environment has a key role to play in the expression of impulsivity, as BPD patients who experience multiple childhood adversities are more likely to have multiple impulsive suicide attempts. ${ }^{82}$ Yet variations in trait impulsivity could also be adaptive or maladaptive depending on circumstances. While it is often better to be cautious than impulsive, it is also possible to be over-cautious: a trait associated with a tendency to rapid action can be adaptive under the right circumstances. Consider, for example, the responses to danger expected from soldiers and police officers, in that effective work requires rapid action. In this case, the tendency for rapid action would be adaptive since the consequences of actions are properly 
considered. Still, even when the consequences of one's actions are not considered, impulsivity can be adaptive. Temperamental impulsivity has been shown to be a susceptibility factor in the prediction of externalizing behaviors, substance use and depressive symptoms. ${ }^{23,33}$ It has been suggested that impulsivity could act as a susceptibility factor because of the novelty-seeking tendency associated with this temperamental trait, which could lead to more developmental opportunities when there is proper guidance from meaningful adults. ${ }^{39}$ Thus, early impulsivity could be expected to have a similar interaction with the environment in the prediction of BPD symptoms.

The third core characteristic of $\mathrm{BPD}$, disturbed relationships, has traditionally been conceptualized as determined by the environment. However, it has been argued in recent years that this characteristic could be central to the disorder and have phenotypic origins ${ }^{83}$ Some early individual characteristics have been suggested as predictors of the disturbed relationships phenotype, and include the serotonergic and dopaminergic systems, as well as distress-prone and more irritable temperaments. ${ }^{84}$ These characteristics need to be researched in the context of BPD as they could interact with the social environment in a differential susceptibility manner when predicting the disturbed relationships phenotype.

The characteristics of people at risk for BPD have been described by evolutionary biologists as a "Hawk" temperamental phenotype, which is an overarching temperament profile characterized by a quick and impulsive approach, an expectation of reward, and tendencies toward irritability or frustration. ${ }^{85}$ This helps us understand why BPD patients are so often attracted to high-stimuli environments. However, the Hawk phenotype is also a marker for differential susceptibility; research shows that children with Hawk-like temperaments show both greater maladjustment with poor quality parenting environments but better psychological 
adjustment with high quality parenting environments. Indeed, it was found that 4.6-year-old children high in the Hawk temperament profile increased more on aggression over 3 years than children low on the Hawk temperament profile when parental intrusive control was high, but increased less on aggression than low-Hawk temperament children when parental intrusive control was low. Similarly, high-Hawk temperament children increased more on depression than low-Hawk temperament children when sensitive parenting was low, but increased less on depression than low-Hawk temperament children when sensitive parenting was high. ${ }^{86}$ Other temperamental traits that have been shown to be associated with BPD and could be looked at as potential susceptibility factors in future research include activity level ${ }^{80}$ and harm avoidance. ${ }^{87}$

While temperament and personality characteristics are most definitely potential susceptibility factors for BPD, gene-environment interactions have also been a focus in differential susceptibility research and could predict BPD symptoms. Indeed, gene-environment interactions have been found to predict BPD in some studies, although the differential susceptibility hypothesis was not tested. Genes interacting with the environment to predict BPD symptoms include the serotonin transporter gene (5-HTTLPR), the brain-derived neurotrophic factor gene (BDNF) and the catechol-O-methyltransferase gene (COMPT), ${ }^{88}$ which have been shown to interact with the environment in a differential susceptibility manner when predicting other developmental outcomes. ${ }^{23,34}$ Beyond temperament and personality traits, physiological reactivity is another interesting marker that could be studied. Notably, lower resting vagal tone has been suggested as a biological vulnerability factor for BPD symptoms because of its association with emotional dysregulation ${ }^{89,90}$ but could serve as a susceptibility factor, especially considering research on anxiety ${ }^{23}$ and aggression ${ }^{91}$ that supported the differential susceptibility model. 
As can be seen, several personal characteristics could potentially act as susceptibility factors for BPD symptoms, making individuals at risk for BPD in adverse environments, but also making them more likely to experience better than average outcomes in positive environments. Indeed, the literature suggests that several temperament traits, including negative emotionality, impulsivity, the Hawk phenotype, activity level and harm avoidance, as well as genes and vagal tone, could be potential susceptibility factors for BPD. Within all the temperament traits identified, harm avoidance and negative emotionality have already been shown to interact with the environment in the prediction of BPD, ${ }^{87,92}$ but these interactions were not fully tested within a differential susceptibility framework.

\section{Testing the Proposed Hypotheses}

Since all the individual characteristics identified in the previous section have not been rigorously tested as susceptibility factors in interaction with the environment to predict BPD symptoms, research examining the differential susceptibility model with BPD is needed. As a first step, previous studies on person-environment interactions could be re-examined to see whether they potentially supported the differential susceptibility model.$^{33}$ While this is a useful first step, this method is more liberal than what can be accomplished by fully analyzing one's data, and thus studies examining the differential susceptibility model a priori would still be needed in a second step. When conducting studies examining differential susceptibility., traditional methodologies used to assess person-environment interactions remain relevant, although ideally one should choose an environmental measure representing the full range of environmental influence, from the negative to the positive. However, statistical analyses will differ, with traditional interaction tests have to be supplemented with additional analyses. 
There are now specific guidelines to test for differential susceptibility using linear regressions to test interaction effects. ${ }^{93}$ These guidelines specify criteria for distinguishing diathesis-stress from differential susceptibility (see Figure 1). The main visual difference between interactions supporting either model concerns the presence or absence of a crossover point, which is a point in the interaction graph where the two regression lines intersect. Support for the diathesis-stress model is obtained when the individual characteristic is associated with the outcome and an ordinal interaction (i.e., without a crossover point; see Figure 1a) is found. In contrast, support for the differential susceptibility model is obtained when the individual characteristic is associated with the outcome and a disordinal interaction (i.e., with a crossover point; see Figure 1b) is found. Furthermore, for both models, the slope of the vulnerable or susceptible group (e.g., individuals high in impulsivity or negative emotionality) has to be significantly different from zero and significantly steeper than the slope of the non-vulnerable or susceptible group (e.g., those low in impulsivity or negative emotionality).

While visual appraisal of the interaction was previously considered sufficient to determine whether the interaction is ordinal or disordinal, further statistical testing is now required to confirm the shape of the interaction. The option used in most studies is to conduct a region-of-significance analysis and the quantification of the proportion of the interaction representing a "for better" effect (see Roisman et al. ${ }^{30}$ and Del Giudice ${ }^{29}$ for details on this approach). More recently, the estimation of the crossover point and its confidence interval has been suggested, ${ }^{31}$ but it has been shown to be unreliable in small samples $(\mathrm{n}<500) .{ }^{94}$ Finally, an alternative to multiple regression is a model-fitting approach, where the differential susceptibility and diathesis-stress models are directly compared without first testing for significant interactions (see Belsky, Pluess \& Widaman ${ }^{24}$ for details). 


\section{Clinical Implications for BPD Treatment}

Finding support for differential susceptibility in BPD would have implications for its clinical conceptualization. Indeed, differential susceptibility is normally used to predict continuous outcomes, i.e., levels of developmental problems or psychopathology. While BPD is currently clinically defined as the presence or absence of a set number of symptoms, the interaction between susceptibility factors and the environment would most likely predict the severity of BPD symptoms, ranging from absent to severe, and not the presence or absence of a BPD diagnosis. Indeed, it would make the most sense for levels of environmental adversity to be associated with varying levels of BPD symptom severity. Research on differential susceptibility and BPD would thus contribute to the definition of the etiology of BPD and to clarify the categorical-dimensional controversy. ${ }^{95}$

Clarifying how individual characteristics interact with the environment to predict BPD symptomatology would allow the development of early prevention programs. As far as we know, pre-pubertal programs to prevent BPD have never been developed, in part because of a lack of research on the developmental risk and protective factors associated with BPD. ${ }^{96,97}$ Research on differential susceptibility would contribute to the identification of these early risk and protective factors. If with such research child temperament or early physiological reactivity is found to be associated with BPD when in an adverse familial environment, parenting or familial interventions could prove effective in reducing the negative effects of this environment, including BPD symptoms. Interventions in child care facilities or schools could also be beneficial since these environments have been shown to interact with temperament in predicting developmental outcomes, ${ }^{41,98-101}$ but their relative importance for such outcomes would need to be compared with the familial environment. Furthermore, identifying children more susceptible 
to their environment and at risk for BPD would allow targeted interventions, which could be useful as universal prevention programs are not considered practical due to the low prevalence of BPD. ${ }^{96}$

The complexity of pathways to BPD shows both multifinality and equifinality. ${ }^{102}$ On the one hand, multifinality implies that different outcomes can result from a same starting point, and thus risk factors associated with BPD can lead to many different forms of psychopathology. On the other hand, equifinality implies that a common outcome can result from different starting points and thus patients who develop BPD can differ in the proportion of interacting genetic and environmental risk factors. One cannot therefore assume that any risk factor must be present simply because it is frequently associated with a clinical diagnosis.

In a clinical setting, keeping the principle of differential susceptibility to the environment in mind can be helpful to clinicians who aim to understand the life histories of these challenging patients. There has been an unfortunate tendency in psychiatry to blame families when BPD develops and to assume that BPD patients must have a history of childhood trauma. The idea that childhood trauma is the main cause of BPD has been influential in clinical settings because it is rather dramatic. ${ }^{103}$ Yet research shows that only a minority of BPD patients have experienced severe childhood trauma, ${ }^{65,103,104}$ and that the effect size of child abuse in relation to developing the disorder is small. ${ }^{105} \mathrm{BPD}$ patients do experience deficits in parenting, but they can be subtle, sometimes involving a well-meaning parenting style that fails to understand a child's emotional needs, leading to a failure to validate emotions. ${ }^{55,106}$ Furthermore, as we have seen, children raised in the same family are not concordant for BPD, even when exposed to similarly adverse parenting. ${ }^{66,67}$ Even if abuse or emotional neglect can be shown to be main effects in a trajectory 
leading to BPD, this may mask interactions with temperamental factors that govern emotional reactions to the environment in individuals.

An evolutionary model of BPD may also be helpful in understanding the mechanisms behind effective therapy. Currently, many treatment methods are based on techniques that modify environmental sensitivity by teaching patients to regulate their emotions. ${ }^{55}$ This approach, based on a differential susceptibility perspective, ${ }^{48}$ can encourage agency and counteract the tendency to attribute psychological problems exclusively to adverse circumstances. The key therapeutic task for BPD patients is to thicken their skin and modulate their reactions to current interpersonal events. While working on BPD patients' susceptibility can be effective, adding an environmental component could also prove useful. Indeed, if a personenvironment interaction predicts the severity of BPD symptoms even after the disorder has been diagnosed, one could expect an improvement in the social environment to be associated with an improvement of BPD symptoms. Thus, the differential susceptibility hypothesis could potentially be an explanatory mechanism for the effectiveness of therapy. Indeed, therapy can influence the environment for the better in several ways. A trusting relation with a therapist can provide a safe environment, allowing the patient to work on improving the social environment, and in making better choices of partners and friends. The differential susceptibility theory could also influence therapists to think interactively about etiological or therapeutic factors instead of thinking in terms of univariate models.

\section{Important considerations for the differential susceptibility theory and concluding remarks}

Although person-environment interaction frameworks suggest that differences in developmental plasticity of behavior are related to genotype, plasticity itself is also influenced by 
the environment. Indeed, plasticity is partly influenced by genes and each individual would have a pre-natally programmed plasticity, with more susceptible individuals presenting more plasticity. However, the degree to which an individual presents plasticity at a specific time in development will be a function of the interaction between this pre-natally programmed plasticity and previous environmental influences. ${ }^{107}$ Thus, there might not only be individual variations in plasticity, but also developmental windows when certain individuals may show greater plasticity. ${ }^{34,108}$

If plasticity can change across development, an important aspect to take into consideration is whether the person-environment interaction proposed here would remain or change across development. Our work showing that temperament likely acts as a susceptibility factor for externalizing behaviors in childhood also suggests that it may be a vulnerability factor supporting diathesis-stress in adolescence. ${ }^{33}$ Thus, examining the differential susceptibility hypothesis to explain the emergence of BPD would also require taking into consideration the developmental period studied. Therefore, and although evidence for differential susceptibility is accumulating, not all person-environment interactions are expected to follow this model. The goal of future research should thus not be proving differential susceptibility over diathesis-stress, but to identify in which circumstances each model applies. Thus, research will have to determine whether diathesis-stress or differential susceptibility best represent interactions between specific personal characteristics and social environments, in the prediction of specific developmental outcomes, and during specific developmental periods.

Finally, as reviewed in this article, person-environment interactions can account for at least part of the development of BPD symptoms, and these interactions may follow a differential susceptibility pattern. However, this remains speculative given the lack of research on the 
subject. Future psychiatric research addressing the diathesis-stress vs. differential susceptibility perspectives will increase our understanding of the etiology of BPD by determining whether early individual risk factors for BPD support the differential susceptibility model in a "for better and for worse" manner. In turn, this will help inform early prevention programs for BPD. 


\section{References}

1. Brune M. Textbook of evolutionary psychiatry and psychosomatic medicine. New York, NY: Oxford University Press; 2015.

2. Jang K. The behavioral genetics of psychopathology: A clinical guide. Mahwah, NJ: Erlbaum; 2005.

3. Plomin R, DeFries JC, Knopik VS, Neiderhiser JN. Bahavioral genetics. New York, NY: MacMillan; 2013.

4. Turkheimer E. Three laws of behavior genetics and what they mean. Curr Dir Psychol 2000;9:160-4.

5. Nesse R, Williams GC. Why we get sick. New York, NY: Penguin; 1993.

6. Belsky J, Bakermans-Kranenburg MJ, van Ijzendoorn MH. For better and for worse:

Differential susceptibility to environmental influences. Curr Dir Psychol 2007;16:300-4.

7. Belsky J, Pluess M. The nature (and nurture?) of plasticity in early human development. Perspect Psychol Sci 2009;4:345-51.

8. Price J, Sloman L, Gardner R, Gilbert P, Rohde P. The social competition hypothesis of depression. Br J Psychiatry 1994;164:309-15.

9. Andreasen NC. Creativity and mental illness: Prevalence rates in writers and their first-degree relatives. Am J Psychiat 1987;144:1288-92.

10. Santosa CA, Strong CM, Nowakowska C, Wang PW, Rennicke CM, Ketter TA. Enhanced creativity in bipolar disorder patients: A controlled study. J Affect Disord 2007;100:31-9.

11. Strong CM, Nowakowska C, Santosa CM, Wang PW, Kraemer HC, Ketter TA. Temperament-creativity relationships in mood disorder patients, healthy controls and highly creative individuals. J Affect Disord 2007;100:41-8. 
12. Simeonova DI, Chang KD, Strong C, Ketter TA. Creativity in familial bipolar disorder. J Psychiatr Res 2005;39:623-31.

13. Costa PT, Widiger TA. Personality disorders and the five factor model of personality. 3rd ed. Washington, DC: American Psychological Association; 2013.

14. Beck AT, Davis DD, Freeman A. Cognitive therapy of personality disorders 3rd ed. New York, NY: Guildford; 2015.

15. Livesley WJ. The practical management of personality disorder. New York, NY: Guildford; 2003.

16. Stearns SC. The evolution of life histories. Oxford, UK: Oxford University Press; 1992.

17. Belsky J, Steinberg L, Draper P. Childhood experience, interpersonal development, and reproductive strategy: An evolutionary theory of socialization. Child Dev 1991;62:647-70.

18. Ellis BJ. Timing of pubertal maturation in girls: An integrated life history approach. Psychol Bull 2004;130:920-58.

19. Brüne M. Life history theory as organizing principle of psychiatric disorders: Implications and prospects exemplified by borderline personality disorder. Psychol Inq 2014;25:311-21.

20. Brüne M. Borderline personality disorderwhy 'fast and furious'? Evolution, medicine, and public health 2016;2016:52-66.

21. Teicher MH, Andersen SL, Polcari A, Anderson CM, Navalta CP, Kim DM. The neurobiological consequences of early stress and childhood maltreatment. Neurosci Biobehav Rev 2003;27:33-44.

22. Teicher MH, Samson JA, Anderson CM, Ohashi K. The effects of childhood maltreatment on brain structure, function and connectivity. Nat Rev Neurosci 2016;17:652-+. 
23. Belsky J, Pluess M. Beyond diathesis stress: Differential susceptibility to environmental influences. Psychol Bull 2009;135:885-908.

24. Belsky J, Pluess M, Widaman KF. Confirmatory and competitive evaluation of alternative gene-environment interaction hypotheses. J Child Psychol Psychiatry 2013;54:1135-43.

25. Monroe SM, Simons AD. Diathesis-stress theories in the context of life stress research: Implications for the depressive disorders. Psychol Bull 1991;110:406-25.

26. Belsky J. Differential susceptibility to rearing influences: An evolutionary hypothesis and some evidence. In: Ellis B, Bjorklund D, eds. Origins of the social mind: Evolutionary psychology and child development. New York, NY: Guildford; 2005:139-63.

27. Simpson JA, Belsky J. Attachment theory within a modern evolutionary framework. In: Cassidy J, Shaver P, eds. Handbook of attachment. 3rd ed. New York, NY: Guildford; 2016. 28. Boyce WT, Ellis BJ. Biological sensitivity to context: I. An evolutionary-developmental theory of the origins and functions of stress reactivity. Dev Psychopathol 2005;17:271-301. 29. Del Giudice M. Statistical tests of differential susceptibility: Performance, limitations, and improvements. Dev Psychopathol 2017:1-12.

30. Roisman GI, Newman DA, Fraley RC, Haltigan JD, Groh AM, Haydon KC. Distinguishing differential susceptibility from diathesis-stress: Recommendations for evaluating interaction effects. Dev Psychopathol 2012;24:389-409.

31. Widaman KF, Helm JL, Castro-Schilo L, Pluess M, Stallings MC, Belsky J. Distinguishing ordinal and disordinal interactions. Psychol Methods 2012;17:615-22.

32. Belsky J, Newman DA, Widaman KF, et al. Differential susceptibility to effects of maternal sensitivity? A study of candidate plasticity genes. Dev Psychopathol 2014:1-22. 
33. Rioux C, Castellanos-Ryan N, Parent S, Séguin JR. The interaction between temperament and the family environment in adolescent substance use and externalizing behaviors: Support for diathesis-stress or differential susceptibility? Dev Rev 2016;40:117-50.

34. Belsky J, Pluess M. Beyond risk, resilience, and dysregulation: Phenotypic plasticity and human development. Dev Psychopathol 2013;25:1243-61.

35. Goldsmith HH, Buss AH, Plomin R, et al. Roundtable: What is temperament? Four approaches. Child Dev 1987;58:505-29.

36. Rothbart MK, Bates JE. Temperament. In: Eisenberg N, ed. Handbook of child psychology: Vol 3 social, emotional, and personality development. 6th ed. Hoboken, NJ: Wiley; 2006. 37. Shiner RL, Buss KA, McClowry SG, Putnam SP, Saudino KJ, Zentner M. What is temperament now? Assessing progress in temperament research on the twenty-fifth anniversary of goldsmith et al. Child Develop Perspect 2012;6:436-44.

38. Thomas A, Chess S. Temperament and development. New York, NY: Brunner/Mazel; 1977. 39. Rioux C, Castellanos-Ryan N, Parent S, Vitaro F, Tremblay RE, Séguin JR. Differential susceptibility to environmental influences: Interactions between child temperament and parenting in adolescent alcohol use. Dev Psychopathol 2016;28:265-75.

40. Slagt M, Dubas JS, Dekovic M, van Aken MAG. Differences in sensitivity to parenting depending on child temperament: A meta-analysis. Psychol Bull 2016;142:1068-110.

41. Essex MJ, Armstrong JM, Burk LR, Goldsmith HH, Boyce WT. Biological sensitivity to context moderates the effects of the early teacher-child relationship on the development of mental health by adolescence. Dev Psychopathol 2011;23:149-61.

42. Aron EN, Aron A. Sensory-processing sensitivity and its relation to introversion and emotionality. J Pers Soc Psychol 1997;73:345-68. 
43. Aron EN, Aron A, Jagiellowicz J. Sensory processing sensitivity: A review in the light of the evolution of biological responsivity. Pers Soc Psychol Rev 2012;16:262-82.

44. Cicchetti D, Rogosch FA, Thibodeau EL. The effects of child maltreatment on early signs of antisocial behavior: Genetic moderation by tryptophan hydroxylase, serotonin transporter, and monoamine oxidase a genes. Dev Psychopathol 2012;24:907-28.

45. Knafo A, Israel S, Ebstein RP. Heritability of children's prosocial behavior and differential susceptibility to parenting by variation in the dopamine receptor $\mathrm{d} 4$ gene. Dev Psychopathol 2011;23:53-67.

46. Daw J, Shanahan M, Harris KM, Smolen A, Haberstick B, Boardman JD. Genetic sensitivity to peer behaviors: 5httlpr, smoking, and alcohol consumption. J Health Soc Behav 2013;54:92108.

47. Brody GH, Beach SRH, Chen YF, et al. Perceived discrimination, serotonin transporter linked polymorphic region status, and the development of conduct problems. Dev Psychopathol 2011;23:617-27.

48. Bakermans-Kranenburg MJ, van IJzendoorn MH. The hidden efficacy of interventions: Gene $\times$ environment experiments from a differential susceptibility perspective. Annu Rev Psychol 2015;66:381-409.

49. Steeger CM, Cook EC, Connell CM. The interactive effects of stressful family life events and cortisol reactivity on adolescent externalizing and internalizing behaviors. Child Psychiat Hum Dev 2017;48:225-34.

50. Obradovic J, Bush NR, Boyce WT. The interactive effect of marital conflict and stress reactivity on externalizing and internalizing symptoms: The role of laboratory stressors. Dev Psychopathol 2011;23:101-14. 
51. Reichborn-Kjennerud T, Ystrom E, Neale MC, et al. Structure of genetic and environmental risk factors for symptoms of dsm-iv borderline personality disorder. JAMA Psychiatry 2013;70:1206-14.

52. Torgersen S, Lygren S, Oien PA, et al. A twin study of personality disorders. Compr Psychiat 2000;41:416-25.

53. Carpenter RW, Tomko RL, Trull TJ, Boomsma DI. Gene-environment studies and borderline personality disorder: A review. Curr Psychiatry Rep 2013;15:7.

54. Caspi A, Moffitt TE. Opinion - gene-environment interactions in psychiatry: Joining forces with neuroscience. Nat Rev Neurosci 2006;7:583-90.

55. Linehan M. Cognitive behavior therapy for borderline personality disorder. New York, NY: Guildford; 1993.

56. Dixon-Gordon KL, Yiu A, Chapman AL. Borderline personality features and emotional reactivity: The mediating role of interpersonal vulnerabilities. J Behav Ther Exp Psychiatry $2013 ; 44: 271-8$.

57. Sauer C, Arens EA, Stopsack M, Spitzer C, Barnow S. Emotional hyper-reactivity in borderline personality disorder is related to trauma and interpersonal themes. Psychiatry Res 2014;220:468-76.

58. Gratz KL, Rosenthal MZ, Tull MT, Lejuez CW, Gunderson JG. An experimental investigation of emotional reactivity and delayed emotional recovery in borderline personality disorder: The role of shame. Compr Psychiat 2010;51:275-85.

59. Brune M. On the role of oxytocin in borderline personality disorder. Br J Clin Psychol 2016;55:287-304. 
60. Hammen C, Bower JE, Cole SW. Oxytocin receptor gene variation and differential susceptibility to family environment in predicting youth borderline symptoms. J Pers Disord $2015 ; 29: 177-92$.

61. Moore SR, Depue RA. Neurobehavioral foundation of environmental reactivity. Psychol Bull 2016;142:107-64.

62. Ruocco AC, Carcone D. A neurobiological model of borderline personality disorder: Systematic and integrative review. Harv Rev Psychiatr 2016;24:311-29.

63. Ripke S, Neale BM, Corvin A, et al. Biological insights from 108 schizophrenia-associated genetic loci. Nature 2014;511:421-+.

64. Paris J. A concise guide to personality disorders. Washington, DC: American Psychological Association; 2015.

65. Zanarini MC. Childhood experiences associated with the development of borderline personality disorder. Psychiatr Clin North Amer 2000;23:89-101.

66. Laporte L, Paris J, Guttman H, Russell J. Psychopathology, trauma, and personality traits in patients with borderline personality disorder and their sisters. J Pers Disord 2011;25:448-62.

67. Laporte L, Paris J, Guttman H, Russell J, Correa JA. Using a sibling design to compare childhood adversities in female patients with bpd and their sisters. Child Maltreat 2012;17:31829.

68. Fok MLY, Hayes RD, Chang CK, Stewart R, Callard FJ, Moran P. Life expectancy at birth and all-cause mortality among people with personality disorder. J Psychosomat Res 2012;73:104-7.

69. Gunnar MR. Early life stress: What is the human chapter of the mammalian story? Child Develop Perspect 2016;10:178-83. 
70. Gratz KL, Roemer L. Multidimensional assessment of emotion regulation and dysregulation: Development, factor structure, and initial validation of the difficulties in emotion regulation scale. J Psychopathol Behav Assess 2008;30:315-.

71. Nock MK, Wedig MM, Holmberg EB, Hooley JM. The emotion reactivity scale:

Development, evaluation, and relation to self-injurious thoughts and behaviors. Behav Therapy 2008;39:107-16.

72. Ebner-Priemer UW, Kuo J, Kleindienst N, et al. State affective instability in borderline personality disorder assessed by ambulatory monitoring. Psychol Med 2007;37:961-70.

73. Russell J, Moskowitz D, Sookman D, Paris J. Affective instability in patients with borderline personality disorder. J Abnorm Psychol 2007;116:578-88.

74. Levenson MR, Aldwin CM, Bosse R, Spiro A. Emotionality and mental health: Longitudinal findings from the normative aging study. J Abnorm Psychol 1988;97:94-6.

75. Meier BP, Robinson MD, Carter MS, Hinsz VB. Are sociable people more beautiful? A zero-acquaintance analysis of agreeableness, extraversion, and attractiveness. J Res Pers 2010;44:293-6.

76. Ellis LK. Individual differences and adolescent psychological development. In: University of Oregon; 2002.

77. Kim S, Kochanska G. Child temperament moderates effects of parent-child mutuality on self-regulation: A relationship-based path for emotionally negative infants. Child Dev 2012;83:1275-89.

78. Raver CC, Blair C, Willoughby M, Family Life Project Key I. Poverty as a predictor of 4year-olds' executive function: New perspectives on models of differential susceptibility. Dev Psychol 2013;49:292-304. 
79. Crockenberg SC, Leerkes EM, Jo PSB. Predicting aggressive behavior in the third year from infant reactivity and regulation as moderated by maternal behavior. Dev Psychopathol 2008;20:37-54.

80. Carlson EA, Egeland B, Sroufe LA. A prospective investigation of the development of borderline personality symptoms. Dev Psychopathol 2009;21:1311-34.

81. Crowell SE, Beauchaine TP, Linehan MM. A biosocial developmental model of borderline personality: Elaborating and extending linehan's theory. Psychol Bull 2009;135:495-510.

82. Soloff PH, Lynch KG, Kelly TM. Childhood abuse as a risk factor for suicidal behavior in borderline personality disorder. J Pers Disord 2002;16:201-14.

83. Gunderson JG. Disturbed relationships as a phenotype for borderline personality disorder. Am J Psychiat 2007;164:1637-40.

84. Gunderson JG, Lyons-Ruth K. Bpd's interpersonal hypersensitivity phenotype: A geneenvironment-developmental model. J Pers Disord 2008;22:22-41.

85. Smith JM, Price GR. The logic of animal conflict. Nature 1973;246:15-8.

86. Hentges RF. Toward greater specificity in identifying the developmental consequences of a risky temperamental phenotype: An evolutionary perspective: University of Rochester; 2015. 87. Arens EA, Grabe HJ, Spitzer C, Barnow S. Testing the biosocial model of borderline personality disorder: Results of a prospective 5-year longitudinal study. Personal Ment Health 2011;5:29-42.

88. Amad A, Ramoz N, Thomas P, Jardri R, Gorwood P. Genetics of borderline personality disorder: Systematic review and proposal of an integrative model. Neurosci Biobehav Rev 2014;40:6-19. 
89. Kuo JR, Linehan MA. Disentangling emotion processes in borderline personality disorder: Physiological and self-reported assessment of biological vulnerability, baseline intensity, and reactivity to emotionally evocative stimuli. J Abnorm Psychol 2009;118:531-44.

90. Koenig J, Kemp AH, Feeling NR, Thayer JF, Kaess M. Resting state vagal tone in borderline personality disorder: A meta-analysis. Prog Neuro-Psychopharmacol Biol Psychiatry 2016;64:18-26.

91. Eisenberg N, Sulik MJ, Spinrad TL, et al. Differential susceptibility and the early development of aggression: Interactive effects of respiratory sinus arrhythmia and environmental quality. Dev Psychol 2012;48:755-68.

92. Haltigan JD, Vaillancourt T. Identifying trajectories of borderline personality features in adolescence: Antecedent and interactive risk factors. Can J Psychiat-Rev Can Psychiat 2016;61:166-75.

93. Frazier PA, Tix AP, Barron KE. Testing moderator and mediator effects in counseling psychology research. J Couns Psychol 2004;51:115-34.

94. Lee S, Lei MK, Brody GH. Confidence intervals for distinguishing ordinal and disordinal interactions in multiple regression. Psychol Methods 2015;20:245-58.

95. Paris J. After dsm-5: Where does personality disorder research go from here? Harv Rev Psychiatr 2014;22:216-21.

96. Chanen AM, McCutcheon LK. Prevention and early intervention for borderline personality disorder: Current status and recent evidence. Br J Psychiatry 2013;202:s24-s9.

97. Chanen AM, McCutcheon LK, Jovev M, Jackson HJ, McGorry PD. Prevention and early intervention for borderline personality disorder. Med J Aust 2007;187:S18-S21. 
98. Belsky J, Pluess M. Differential susceptibility to long-term effects of quality of child care on externalizing behavior in adolescence? Int J Behav Dev 2012;36:2-10.

99. Pluess M, Belsky J. Differential susceptibility to rearing experience: The case of childcare. J Child Psychol Psychiatry 2009;50:396-404.

100. Pluess M, Belsky J. Differential susceptibility to parenting and quality child care. Dev Psychol 2010;46:379-90.

101. Mortensen JA, Barnett MA. Teacher-child interactions in infant/toddler child care and socioemotional development. Early Education and Development 2015;26:209-29.

102. Cicchetti D, Rogosch FA. A developmental psychopathology perspective on adolescence. J Consult Clin Psychol 2002;70:6-20.

103. Gunderson JG. Borderline personality disorder: Ontogeny of a diagnosis. Focus 2010;8:230-9.

104. Paris J, Zweigfrank H, Guzder J. Psychological risk factors for borderline personality disorder in female patients. Compr Psychiat 1994;35:301-5.

105. Fossati A, Madeddu F, Maffei C. Borderline personality disorder and childhood sexual abuse: A meta-analytic study. J Pers Disord 1999;13:268-80.

106. Marcoux A-A, Bernier A, Séguin JR, Boike Armerding J, Lyons-Ruth K. How do mothers with borderline personality disorder mentalize when interacting with their infants? Personal Ment Health 2017;11:14-22.

107. Pluess M, Stevens S, Belsky J. Differential susceptibility: Developmental and evolutionary mechanisms of gene-environment interactions. In: Legerstee M, Haley DW, Bornstein MH, eds. The infant mind: Origins of the social brain. New York, NY: Guildford Press; 2013:77-96. 
108. Ellis BJ, Boyce WT, Belsky J, Bakermans-Kranenburg MJ, van Ijzendoorn MH.

Differential susceptibility to the environment: An evolutionary-neurodevelopmental theory. Dev Psychopathol 2011;23:7-28. 
a. Diathesis-stress

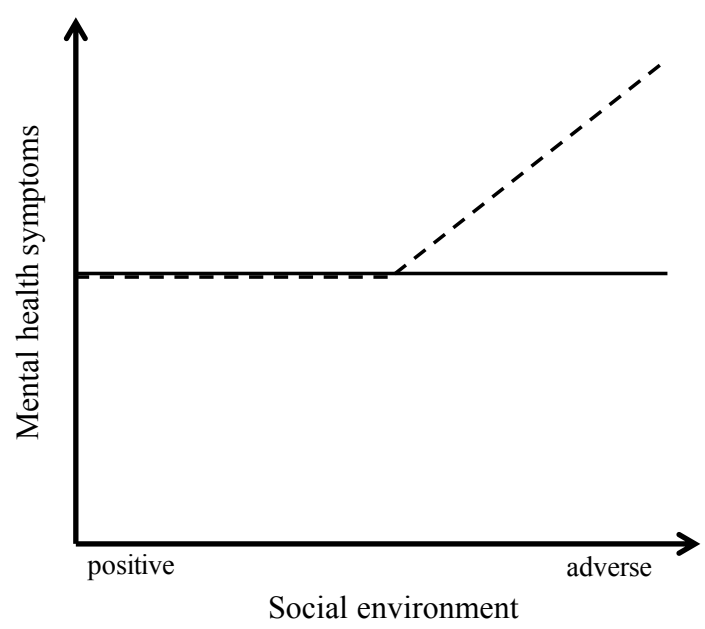

b. Differential susceptibility

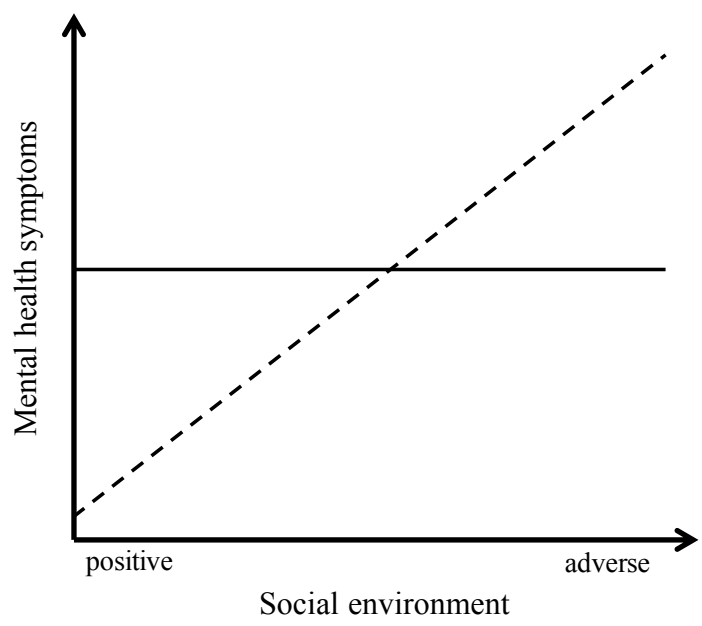

Figure 1. Graphical representation of (a) the diathesis-stress model and (b) the differential susceptibility model. The continuous line represents variations in mental health across environments for resilient traits, and the dashed line represents vulnerability or susceptibility traits. 